\title{
2. On the Behaviours of Enormously Large Internal \\ Stress accompanying the Transformation of a Substance.
}

\author{
By Kotaro HondA, M.J.A. \\ (Comm. Jan. 12, 1949.)
}

I. Introduction.

For the sake of easy explanation, the ease of $A_{3}$ transformation in iron will be taken into discussion, but a similar reasoning will also apply to other cases of transformation or the change of states.

The $A_{3}$ transformation of pure iron is depressed as the content of nickel in iron increases, at first rapidly and then somewhat slowly down to 30 per cent ot nickel. The $A_{3}$ transformation-concentration curve differs greatly during heating and cooling, and, the heating curve lies considerably above the cooling curve, as shown in Fig. 1. Each of them has a range of transformation, which is not, however, shown in the figure ${ }^{1)}$. The cause of this large irreversibility has not yet been explained satisfactorily, but the present writer considers it to be due to the presence of enormously large tension or pressure accompanying to the volume change during the transformation $\alpha \rightarrow \gamma$ or $\gamma \rightarrow \alpha$ respectively.

Supposing that these changes begin from several centres of nuclei and radually grow up to crystal grains, the ransformation $\alpha \rightarrow \gamma$ is accompanied by a contraction or by a tension from its surrounding, while the change $\gamma \rightarrow \alpha$ undergoes an expansion or a pressure, so

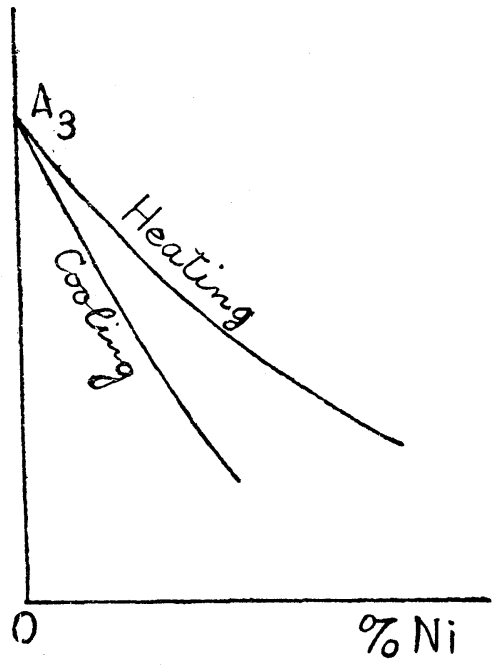

Fig. $1 \quad \mathrm{~F}_{2}-\mathrm{Ni}$ Alloys that by Le Chatelier's principle, the temperature of the change will be lowered by the pressure from its surrounding, the maximum value of which may amount to several thousand atmospheres; but the temperature of the change $\alpha \rightarrow \gamma$ will be raised by the tension of the same order of magnitude as above, from its surround-

1) K. Honda and S. Miura, Sci. Rep. 16, p. 745 (1929). 
ing. Fig. 2 shows a dilatation curve of $10 \% \mathrm{Ni}$ alloy, in which large discontinuities and irreversibility of $120^{\circ} C$, during heating and cooling, are observed. In this figure, $A A^{\prime}$ and $B B^{\prime}$ are the change of length during heating and cooling transformations, respeetively, and $C C^{\prime}$ is the supposed change of length, when the tension or pressure does not exist at all. With falling

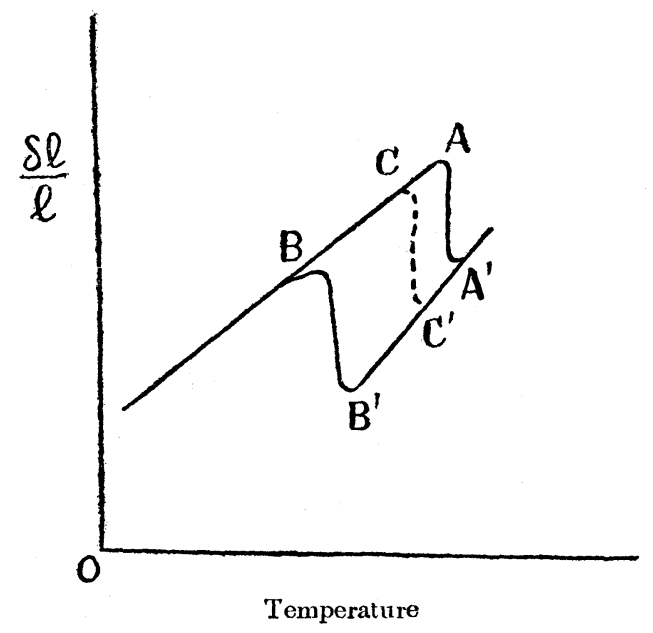

Fig. 2 10\% Ni alloys temperature, $\gamma$ phase remains initially unchanged, until the temperature $C^{\prime}$ is reaehed, where by virtue of the transformation force, the change should begin to occur, but it is prevented by the pressure to do so and hence at a certain lower temperature $B^{\prime}$, the former prevails over the latter force, and the change $\gamma \rightarrow \alpha$ begins actually to take place. During heating the reverse changes will take place, and so by a cyclic change of heating and cooling, a large hysteresis loop will be obtained, the stored energy being assumed to be dissipated in the surrounding media after each cycle of transformation.

II. Calculation of Tension and Pressure caused by Transformation.

As already remarked, a transformation actually starts from several centres of nuclei, and their size gradually grows up isothermally, but for the sake of simplicity of calculation, it is assumed that the transformation $\gamma \rightarrow \alpha$ starts from a single centre of nucleus, as shown in Fig. 3, and that the inner sphere is $\alpha$ iron and the enter one $\gamma$ iron; then the same figure may be taken as representing any stage of $\gamma \rightarrow \alpha$ transformation. If $r_{1}, r_{2}$ are the radii of two concentric spheres, and $p_{1}, p_{2}$ the pressures on the surfaces, of these spheres, respectively, then by the theory of elasticity ${ }^{2}$ it

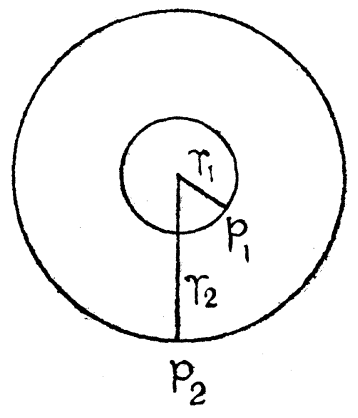

Fig. 3 point $r$ and the pressure $p$ at the same point are given as follows:-

2) T. Tamaru, Iwanami Koza, Rikigaku II, p. 226. 


$$
\begin{aligned}
\delta \gamma_{1} & =C_{1} r_{1}+\frac{C_{2}}{r_{2}^{2}} \\
p_{1} & =-3 K C_{1}+\frac{4 n}{r_{2}^{2}} C_{2}
\end{aligned}
$$

where

$$
\begin{aligned}
& C_{1}=\frac{p_{1} r_{1}^{3}-p_{2} r_{2}^{3}}{3 K\left(r_{2}^{3}-r_{1}^{3}\right)} \\
& C_{2}=\frac{\left(p_{1}-p_{2}\right) r_{1}^{3} r_{2}^{3}}{4 n\left(r_{2}^{3}-r_{1}^{3}\right)}
\end{aligned}
$$

$K$ being the bulk modulus and $n$ the rigidity. If Poisson ratio is taken to be $\frac{1}{3}$, then

$$
K=\frac{8}{3} n \quad \text { and } \quad E=K
$$

So the change of volume of the inner sphere per unit of volume becomes finally

$$
\frac{\delta v}{v}=-\frac{R}{K}+\left(\frac{3}{4 n}+\frac{1}{K}\right)\left(p_{1}-p_{2}\right) \frac{r_{2}^{3}}{r_{2}^{3}-r_{1}^{3}}
$$

If in the above expression, $p_{2}$ is very small as compared to $p_{1}$, then

$$
\frac{\delta v}{v}=\frac{2 p_{1}}{K} \frac{\left(1+\frac{r_{1}^{3}}{2 r_{2}^{3}}\right)}{\left(1-\frac{r_{1}^{3}}{r_{2}^{3}}\right)}
$$

hence

$$
p_{1}=p=\frac{1}{2} K \frac{\delta v}{v} \frac{\left(1-\frac{r_{1}^{3}}{r_{2}^{3}}\right)}{\left(1+\frac{r_{1}^{3}}{2 r_{2}^{3}}\right)}
$$

If $r_{2}$ is very large as compared to $r_{1}$, then

$$
p=\frac{1}{2} K \frac{\delta v}{v}
$$

From the above relations, it can be seen that the pressure $p$ for different values of $r_{1}$ does not remain constant, but it decrease at first slowly and then rapidly, tending to zero at $r_{1}=r_{2}$, that is, when the whole mass is just transformed from $\gamma$ to $a$ iron, as shown in Fig. 4.

The mean intensity of pressure during a complete transformation can be found by a simple integration to be 


$$
\begin{aligned}
\bar{p} & =\int_{0}^{1} p d r^{\cdot}=\frac{1}{2} K \frac{\delta v}{v} \int_{0}^{1} \frac{\left(1-\frac{r_{1}^{3}}{r_{2}^{3}}\right)}{\left(1+\frac{r_{1}^{3}}{2 r_{2}^{3}}\right)} \\
& =0.355 K \frac{\delta v}{v}
\end{aligned}
$$

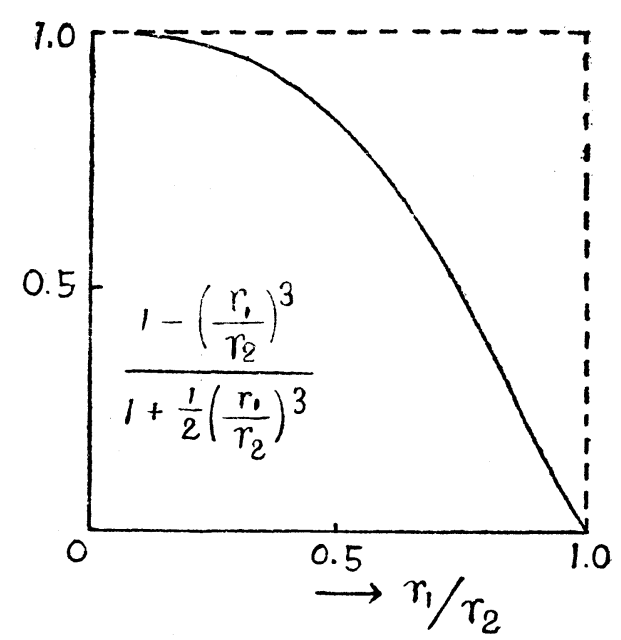

Fig. 4

The mean intensity of pressure is thus obtained; it is only short of $30 \%$ from the maximum value above obtained, that is,

$$
p=\frac{1}{2} K \frac{\delta v}{v}
$$

\section{Clausius-Clapeyron's Equation.}

Clausius-Claperon's equation, which gives the change of transformation point $\Delta T$ caused by applied hydrostatic tension or pressure $p$, is as follows:-

$$
\Delta T=T \frac{\delta v}{v} p / \rho \lambda
$$

where $T, \frac{\delta v}{v}, p, \rho \lambda$ are the transformation point, the volume change, the density, and the latent heat of transformation, respectively. If in place of the external pressure or tension $p$, the internal stress $\bar{p}$ called forth in the transformation system (Fig. 3) can be substitute for $p$, then the change off transformation point by stress can be calculated by means of Clausius-Clapeyron's equation above refered to. 
In the following pages it will be shown that the agreement between the calculated values and the observed ones is in general very satisfactory, confirming the correctness of the above eonsiderations.

From two rclations

$$
p=0.355 \frac{\delta v}{v}
$$

and

$$
\Delta T=T \frac{\delta v}{v} p / \rho \lambda
$$

we have

$$
\Delta T=0.355 K T\left(\frac{\delta v}{v}\right)^{2} / \rho \lambda
$$

Thus it is seen that $\Delta T$ is a square function of $\frac{\delta v}{v}$ and therefore independent of its sign. Again, if $\frac{\delta v}{v}$ is positive, $p$ or $\bar{p}$ shows a pressure, and if it is negative a tension; during heating, $\lambda$ is always positive and during cooling it is negative. Thus the sign of $\Delta T$ depends solely on that of $\lambda$.

If in the exprassion for $\Delta T$ the suffixed letters $e$ and $r$ represent quantities belongiug to heating and cooling stages, the following expressions give $\Delta T_{c}$ and $A T_{r}$ reckoned from the case of vanishing internal stress:

$$
\Delta T_{c}=0.355 K_{c} T_{c}\left(\frac{\delta v}{v}\right)_{c}^{2} / \rho \lambda
$$

and

$$
\Delta T_{r}=0.355 K_{r} T_{r}\left(\frac{\delta v}{v}\right)_{r}^{2} / \rho \lambda
$$

IV. Pure Iron.

In the case of $A_{s}$ transformation of pure iron, $\frac{\delta v}{v}$ and other quantities may be taken as follows:-

$$
\begin{aligned}
& \frac{\delta v}{v}=8.46 \times 10^{-3} \\
& n=0.25 \times 10^{12} \\
& K=\frac{8}{3} n \\
& \rho=7.8 \\
& \lambda=5.6 \times 4.2 \times 10^{7}
\end{aligned}
$$

From these values of constints, $p, \Delta T c, \Delta T r$ can be calculated; thus 
Max. pressure: $\quad p=\frac{1}{2} K \frac{\delta v}{v}=2820$ Atm. pressures

Mean pressure: $\quad \bar{p}=0.71 p=2000 \mathrm{Atm}$. pressures

In the case of pure iron, the difference of $A c_{3}$ and $A r_{3}$ joints is very small, so that the following relations exist, that is,

$$
K_{c} \fallingdotseq K_{r}, \quad T_{c} \fallingdotseq T_{r}, \quad\left(\frac{\delta v}{v}\right)_{c} \fallingdotseq\left(\frac{\delta v}{v}\right)_{r}
$$

Hence

or

$$
\begin{aligned}
\Delta T & =\left|\Delta T_{c}\right|+\left|\Delta T_{r}\right|=0.355 K T\left(\frac{\delta v}{v}\right)^{2} / \rho \lambda \\
& =22.0^{\circ} \mathrm{C}
\end{aligned}
$$

$$
\Delta T_{c}=-\Delta T_{r}=11.0^{\circ} \mathrm{C}
$$

Thus the total change of $A_{3}$ transformation during heating and cooling becomes $22.0^{\circ} \mathrm{C}$, while the observed value is about $20^{\circ} \mathrm{C}$; the agreement is very satisfactory. Such a small irreversibility usually observed during $A c_{3}$ and $A r^{3}$ transformations is so common to us that'one could not throw any doubt about its presence, but according to the above considerations, this irreversibility is caused by the tension and the pressure due to the changes $\alpha \rightarrow \gamma$ and $\gamma \rightarrow \alpha$, respectively.

\section{Iron-Nickel Alloys.}

In iron alloys containing a certain amount of nickel both $\frac{\delta v}{v}$ and $K$ increase as the temperature falls; this can be seen from dilatation and rigidity-temperature curves of these alloys. The following table contains observed data, the quantities belonging to pure iron being taken as unit.

Volume change

Transformation point

Bulk Modulus

\begin{tabular}{l|l|l|ll|l|l|ll|l|l|l}
\hline Alloys & 0 & 10 & $20 \% \mathrm{Ni}$ & Alloys & 0 & 10 & $20 \% \mathrm{Ni}$ & Alloys & 0 & 10 & $20 \% \mathrm{Ni}$ \\
\hline$(\delta v / v)_{r}$ & 1 & 1.85 & 3.9 & $T_{r} / T_{o}$ & 1 & 0.627 & 0.409 & $K_{r}$ & 1 & 2.721 & 3.08 \\
$(\delta v / v)_{c}$ & 1 & 1.50 & 1.6 & $T_{c} / T_{o}$ & 1 & 0.818 & 0.733 & $K_{c}$ & 1 & 1.72 & 2.24 \\
\hline
\end{tabular}

In the above table, the letters $r, c$ suffixed to the quantities denote as before the same quantities in cooling and heating stages, respcctively, also numbers 0 , 10,20 and $x$ in general, suffixed to the quantities $\frac{\delta v}{v}, K, T$ denote the concentrations of nickel in these specimens. Then for the total change of $A_{3}$ transformation point during heating and cooling which is the sum of depressed

3) T. Kikuta, Sci. Rep. 10 (192i), p. 68. 
and elevated temperatures, we have the following expression;

$$
\Delta T_{x}=\Delta T_{0} \frac{T_{r x} K_{I x}\left(\frac{\delta v}{v}\right)_{I x}^{2}+T_{c x} K_{c x x}\left(\frac{\delta v}{v}\right)_{c x}^{2}}{2 T_{0}^{\prime} K_{0}\left(\frac{\delta v}{v}\right)_{0}^{2}}
$$

The quantities found in the right hand side of the above expression being all known, the total change of the transformation point can be calculated; so we get

\begin{tabular}{c|c|c|c|c}
\hline$\Delta T_{x}$ & $\Delta T_{r x}$ & $\Delta T_{c x}$ & Sum & Obs. values \\
\hline$\Delta T_{0}$ & $11.0^{\circ} \mathrm{C}$ & $11.0^{\circ} \mathrm{C}$ & $22 .{ }^{\circ} \mathrm{C}$ & $20.0^{\circ} \mathrm{C}$ \\
$\Delta T_{10}$ & 64.1 & 34.8 & 98.9 & 119.0 \\
$\Delta T_{20}$ & 210.3 & 46.2 & 256.5 & 297.0 \\
\hline
\end{tabular}

Thus the theoretical and observed values are in a good agreement with cach other, proving that Clausius-Clapyron's thermodynamical relation as well as the expression for $p$ or $\bar{p}$ holds good in the case of transformation of iron-nickel alloys. In this calculation the variation of $\lambda$ due to concentrations of nickel was not taken into considerations. Fig. 5 shows the relations of $\Delta T_{r}$ and $\Delta T_{c}$ to the concentration of nickel; the sum of the ordinates for each alloy is the total change of transformation point of the specimens.

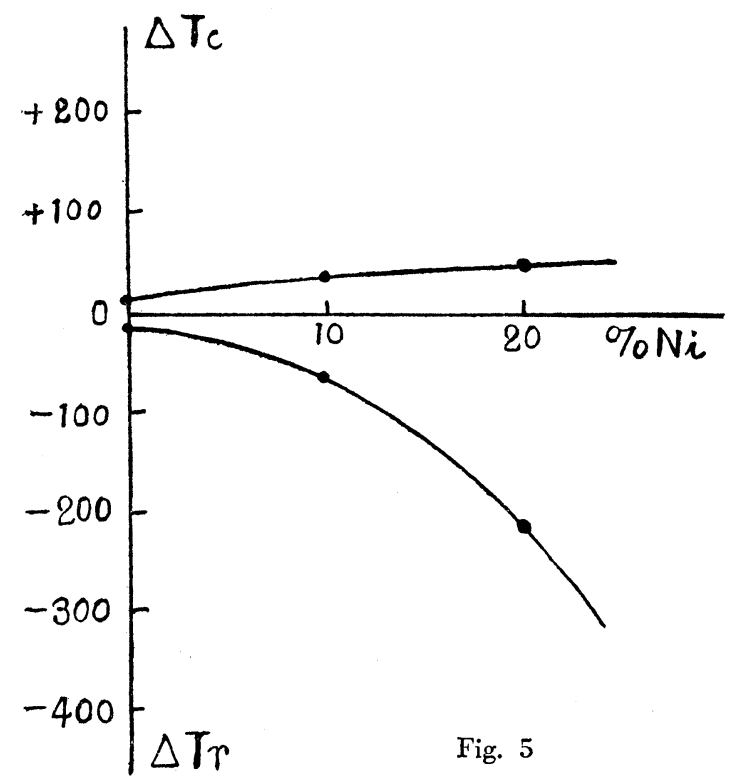

The above theory so far discussed explains also the origin of the out-coming of reversible and irreversible alloys in the binary system of iron and nickel; thus 
the irreversibility of the alloys below $30 \% \mathrm{Ni}$ is caused by the effect of tension and pressure during $A_{3}$ transformation, while the reversibility of other alloys of the same system is explained by the absence of this transformation, as well as.by the smallness of $\frac{\delta v}{v}$ duriug magnetic transformation.

\section{Iron-Manganese Alloys.}

In the case of Fe-Mn alloys of low percentages, the matter is nearly the same as that of iron-nickel alloys so far discussed, the difference being only in the values of $K, T, \frac{\delta v}{v}$.

Through an elaborate investigation, Professor T. Ishiwara ${ }^{4)}$ obtained an equilibrium diagram of iron-manganese system as shown in Fig. 6 . In the iron

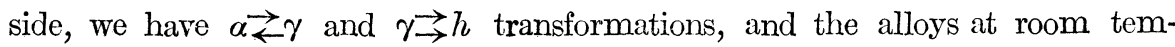
perature consist of $\alpha, \alpha^{\prime}$ (martensite), $h$ and $\gamma$ phases, the latter two being only feebly magnetic. In ordinary rate of heating or cooling, manganese atoms within iron lattice ean not, however, take their equilibrium positions by virtue of the slowness of diffusion, and so the $A_{3}$ lines during heating or cooling, like in

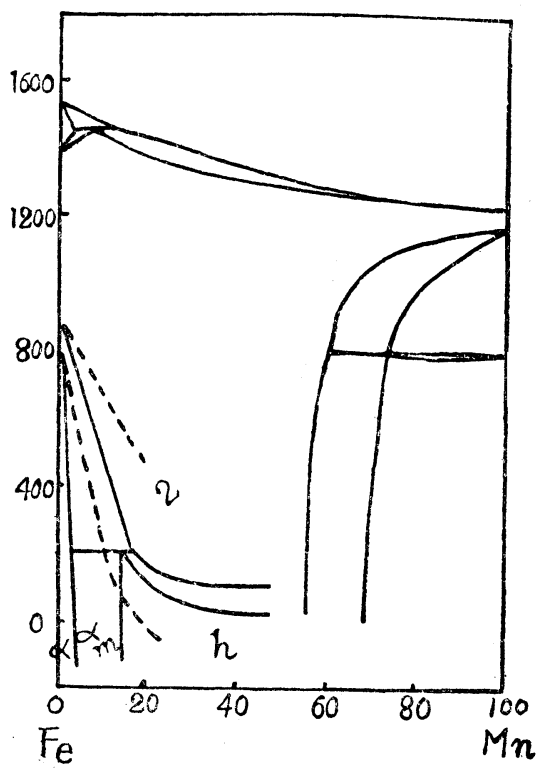

Iron-Manganese

Fig. 6 the case of iron-nickel alloys, fall rapidly with increasing content of manganese, as shown by dotted lines in Fig. 6.

If manganese content further increases, these alloys of solid solution partially decomposes at low temperatures, giving rise to feebly magnetic h-phase, the amount of which increases with manganese content. This can be realized by comparing dilatationtemperature and magnetization-temperature curves given by Professor Ishiwara. Hence in the comparison of the values of $\frac{\delta v}{v}$ for diffcrent manganese alloys, the above tact must be taken into account. So the above theory of the change of the $A_{3}$ transformation point due to pressure and tension

4) T. Ishiwara, Sci. Rep. 19 p. 499 (1930). 
can be applied to the case of solid solution of iron-manganese alloys of low percentages.

The following table contains data necessary for the calculation of the clevation and depression of transformation point by tension and pressure, respectively. The results of calculation for $\Delta T x$ are also included in the same table, and are compared with the observed values.

Table 3

\begin{tabular}{l|l|l|l|l|l|l|l}
\hline$\% M_{n}$ & $K_{r} / K_{0}$ & $K_{c} / K_{0}$ & $T_{r} / T_{0}$ & $T_{c} / T_{0}$ & $\left(\begin{array}{c}\delta v \\
v\end{array}\right)_{r}^{2}$ & $\left(\frac{\delta v}{v}\right)_{c}^{2}$ & Mass-factor \\
\hline 0 & 1 & 1 & 1 & 1 & 1 & 1 & 1 \\
1.0 & 1.44 & 1.61 & 0.860 & 0.918 & 2.25 & 2.25 & 0.765 \\
1.8 & 2.68 & 1.44 & 0.625 & 0.887 & 5.30 & 1.96 & 0.593 \\
3.4 & 2.88 & 1.44 & 0.538 & 0.860 & 5.90 & 2.78 & 0.480 \\
\hline
\end{tabular}

Table 4

\begin{tabular}{l|c|c|c}
\hline$\% \mathrm{Nn}$ & $\Delta T_{r x}+\Delta T_{c x}$ & Sum & Obs. values \\
\hline 0 & $11.0+11.0$ & 22 & 20 \\
1.0 & $38.4+40.2$ & .75 & 66 \\
1.8 & $167.0+47.4$ & 214.4 & 233 \\
3.4 & $206+78.7$ & 284.7 & 324 \\
\hline
\end{tabular}

In table $4, \Delta T$ is the values calculated by the formulae

$$
\Delta T=\Delta T_{0} \frac{T_{r} K_{r}\left(\frac{\delta v}{v}\right)_{r}^{2}+T_{c} K_{c}\left(\frac{\delta v}{v}\right)_{c}^{2}}{2 T_{0} K_{0}\left(\frac{\delta v}{v}\right)_{0}^{2} \text { (mass-factor) }}
$$

Here the mass-factor means a number, by which the values of $\frac{\delta v}{v}$ of the transforming alloys are reduced to the same mass; since the magnitude of the intensity of saturation magnetization is proportional to the mass of the magnetic alloys, the relative mass of the transformed $a$ phase will be given by taking the ratio of the intensities of saturation magnetization of respective alloys as regard to pure iron. The numbers given in the column under heading " mass-factor" are deduced from Professor Ishiwara's magnetic data, by taking the squires of their ratios. As seen from the above table, the agreement between the theory and the experiment is very satisfactory, if we consider the circumstance of smallness of the mass-factor of the alloys of high manganese content. The relation of $\Delta T r$ and $\Delta T c$ to the concentration of manganese is given in Fig. 7 ; the general form 
of the curves are similar to those of Fig. 6.

In the region containing higher percentages of manganese lying between 15-40\%, another transformation $l \rightleftarrows \gamma$ is observed in the dilatometric measurement as an expansion during heating and a contraction during cooling, thereby the temperature-hysteresis amounting to over $150^{\circ} \mathrm{C}$. It is here to be remarked

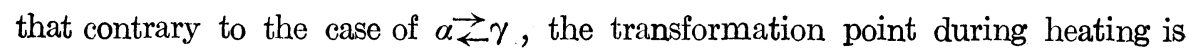
raised and that during cooling lowered, so as to form a large hystereis loop, by the action of pressure or tension, respectively.

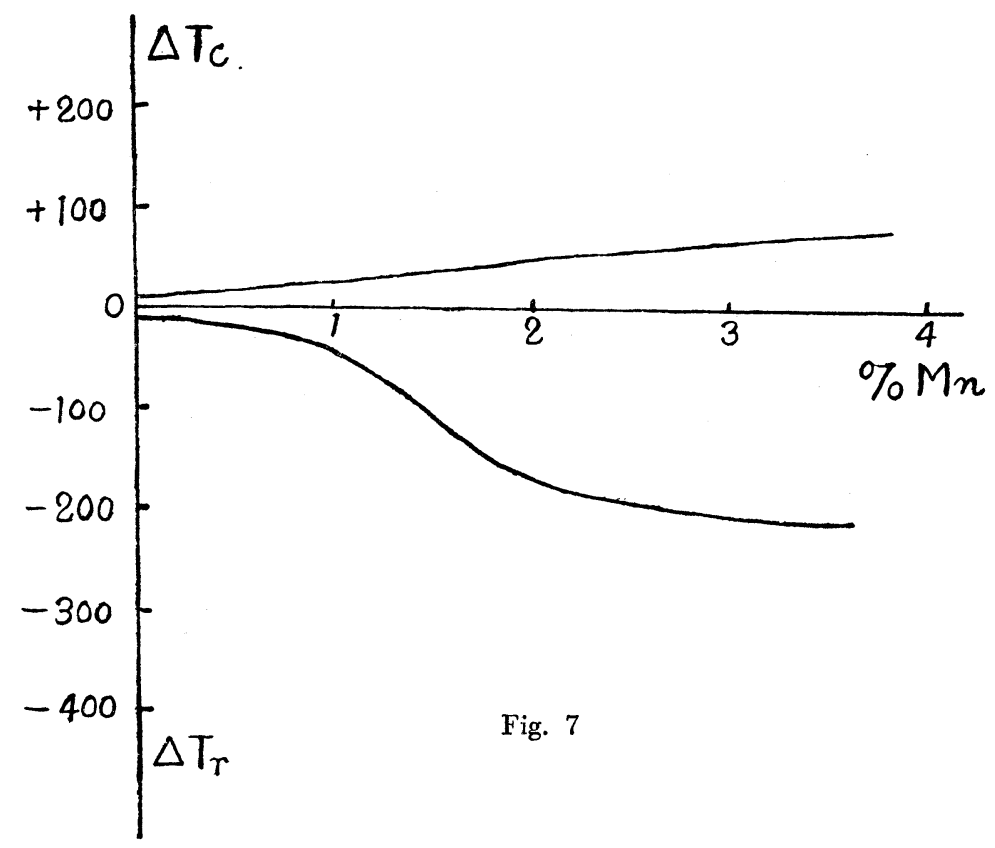

VII. Water.

In Fig. 2, assume the inner sphere to be water and the outer one ice; then this case is quite analogous to that of $\gamma \rightarrow \alpha$ transformation in iron, freezing beginning from outside towards inside. Taking the following values of constants

$$
\begin{array}{ll}
p=0.355 K \frac{\delta v}{v} & \dot{\delta}=0.91 \\
K=1.03 \times 10^{\prime \prime} & T=273 \\
\lambda=80 \times 4-2 \times 10^{i} & \frac{\delta v .}{v}=0.09
\end{array}
$$

we get finally

$$
\begin{aligned}
& \bar{p}=3280 \text { Atm. Pressure. } \\
& \Delta T=-26.4^{\circ} \mathrm{C}
\end{aligned}
$$


According to the results of observation by Bridgman and others, the depression of the freezing point of water by hydrostatic pressure has been confirmed to be $22^{\circ} \mathrm{C}$ under a pressure of 2022 Atms. and increases linearly with the pressure; hence unter 3280 Atms., it will becomes $-32.8^{\circ} \mathrm{C}$; this value coincides fairly well with the theoretical one.

So far the good agreement between the theory and the experiments proves the validity of expressions for the internal pressure. If so, the depression or elevation of a transformation point can be obtained from the simple measurements of $\mathrm{T}, \mathrm{K}$ and $\frac{\delta v}{v}$ during the transformation. This new method of measuring high pressurc effect on the transformation point is very simple and convenient, because it does not require any complicated apparatus and arrangements; notwithstanding it possesses a certain high degree of accuracy. This method can also be conveniently used in the determination of depression of freezing point for different liquids, as well as for metals and alloys, by pressure.

The present investigation so far explained in the above starts from a quite different view-point from that made by Professors K. Iwase, S. Takeuchi"i), and others; but it reveals an important fact that if a substance undergoes a transformation accompanied by a volume change during heating or cooling, a large internal stress is called forth and several properties of the substance are affected by it. So in considering the change of physical properties of a substance during transformation, this stress should be taken into account.

In conclusion, the present writer wishes to express his hearty thanks to Professors T. Hironé, M. Yamada, and Lecturer Y. Chino for their constant advice and assistance during the last one and half years of investigation of this important problem.

5) Nippon Kinzokugakkai-shi, 5 (1911), p. 686 (1942), p. 361. 